\title{
La incapacidad permanente en una empresa pública en el periodo 1995-2009
}

\author{
Permanent incapacity in a public company from 1995-2009
}

\author{
José Carlos Marchena Aparicio ', Cristina O’Ferrall González², Antonio Ares Camerino ', Mª Eugenia González \\ Domínguez $^{3}$, José Manuel Romero Sánchez ${ }^{3}$
}

1. Servicio de Prevención y Salud Laboral. Diputación Provincial de Cádiz. Cádiz. España.

2. Departamento de Enfermería y Fisioterapia. Facultad de Enfermería y Fisioterapia. Universidad de Cádiz. Cádiz. España.

3. Servicio de Prevención y Salud Laboral del Centro Bahía de Cádiz de Airbus Military EADS. El Puerto de Santa María (Cádiz). España.

Recibido: 26-07-11

Aceptado: 09-08-11

\author{
Correspondencia \\ José Carlos Marchena Aparicio \\ Servicio de Prevención y Salud Laboral \\ Diputación de Cádiz. Edificio Roma \\ Avda. Ramón de Carranza s/n \\ 11071 Cádiz. España. \\ Tfno: 956240681 \\ e-mail: jcmarchena@dipucadiz.es
}

Resumen

Objetivos: La cuantificación de las relaciones entre salud, medio laboral y capacidad para el trabajo nos permite descifrar aspectos importantes de la incapacidad permanente. Este trabajo tiene como objetivo describir las características de la población trabajadora de la Diputación Provincial de Cádiz a la que se le concedió una incapacidad permanente en el periodo 1995-2009.

Métodos: Se llevó a cabo un estudio descriptivo retrospectivo sobre el total de trabajadores/as con incapacidad permanente en el periodo descrito que considera las siguientes variables: sexo, edad, antigüedad, tipo de ocupación, causa de la incapacidad permanente, días de incapacidad temporal y número de incapacidades temporales concedidas en los 5 años previos, recogidas a través de archivos documentales.

Resultados: El número de incapacidades permanentes concedidas ascendió a 192. Un 53,1\% fueron mujeres; funcionarios el 59,9\%; la edad de concesión fue de 54,06 años de media con 24,98 años de antigüedad en la empresa; el tipo de trabajo en el que más incapacidad permanente se concedieron es el manual, 44,8\%; El tipo de patología más frecuente de incapacidad permanente fueron las enfermedades musculo-esqueléticas $(29,7 \%)$, neoplasias $(23,4 \%)$ y trastornos mentales $(18,8 \%)$, asociándose significativamente dicha variable con el sexo $(\mathrm{p}=0,006)$, el tipo de trabajo desempeñado $(\mathrm{p}=0,007)$, la edad de concesión $(\mathrm{p}<0,001)$ y el grado asignado $(\mathrm{p}<0,000)$.

Conclusiones: Este estudio aporta indicios sobre la frecuencia y distribución de factores sociodemográficos y propios del proceso de incapacidad permanente que permiten ir descifrando algunos aspectos susceptibles de ser abordados desde el punto de vista de la prevención.

Med Segur Trab (Internet) 2011; 57 (224) 256-264

Palabras clave: Ausencia por Enfermedad; Seguro por Discapacidad; Evaluación de la Discapacidad; Gobierno Local. 


\section{Abstract}

Objectives: The quantifying relationship between health, working environment and work capacity allows us to unravel important aspects of permanent incapacity (PI). One of the objectives of this study is to describe working population characteristics of the Provincial Council of Cadiz who were granted permanent incapacity from 1995 to 2009.

Method: A retrospective descriptive study was carried out amongst all the workers with PI in the period described with the following variables taken into account: gender, age, time working for the company, type of occupation, cause of PI, temporary work incapacity (TWI) days and number of TWI in the last 5 years that have been recorded in the company's archives.

Results: The number of PI granted to workers was 192, 53,1\% of which were women; 59,9\% officials. The average age of workers who were granted PI was 54,06 years having worked in the company for an average of 24,98 years. Within the company most PIs' were manual labor, $44,8 \%$; the most frequent type of pathology for PI was musculoskeletal $(29,7 \%)$ followed by neoplasia $(23,4 \%)$ and mental diseases $(18,8 \%)$, the last one in particular was associated to gender $(p=0,006)$, the type of work done $(p=0,007)$, the age of PI acceptance $(\mathrm{p}<0,001)$ and the grade assigned $(\mathrm{p}<0,000)$.

Conclusion: This study shows us signs regarding the frequency and the distribution of social demographic factors as well as signs belonging to the PI process. This enables us to see some key aspects to be taken into account from the point of view of prevention.

Med Segur Trab (Internet) 2011; 57 (224) 256-264

Keywords: Sick Leave; Disability Insurance; Disability Assessment; Local Government. 


\section{INTRODUCCIÓN}

La Incapacidad Permanente (IP) es la consecuencia final de un proceso patológico o traumático derivado de una enfermedad o accidente en el que, después de haber intentado todos los recursos terapéuticos, no se ha conseguido recuperar un nivel de capacidad funcional compatible con el desarrollo de actividades laborales ${ }^{1}$, lo que trae como consecuencia la anulación o disminución de la capacidad de trabajo.

La IP está condicionada por la normativa existente en cada país, por la situación socio-laboral y por otras circunstancias ajenas al proceso de enfermar. En España existe una normativa específica para la evaluación y calificación de las solicitudes de Incapacidad Permanente por parte de las Direcciones Provinciales del Instituto Nacional de la Seguridad Social.

Los límites legales máximos y mínimos de esa capacidad disminuida están bien delimitados y vinculados estrechamente con la incidencia que, para la profesión habitual o para todo trabajo, tienen las lesiones o dolencias que determinan la incapacidad. Según esto se dividen en los grados de incapacidad permanente parcial para la profesión habitual, incapacidad permanente total para la profesión habitual, la incapacidad permanente absoluta para todo trabajo y la gran invalidez cuando se necesita además la asistencia de otra persona para los actos más esenciales de la vida ${ }^{2}$.

La concesión de estas incapacidades supusieron un 10,79\% de las pensiones contributivas en 2009 (920.900), lo que ha generado un gasto para la Seguridad Social de $831,49 €$ mensuales de media por cada incapacidad concedida ${ }^{3}$. El 96,4 \% de éstas fueron calificadas como incapacidades totales (503.280) y absolutas (384.490), cifra sensiblemente superior al año anterior.

Esta progresión ascendente del número de pensiones por incapacidad permanente, genera cierta inquietud al hacerse patente que el sistema actual de pensiones es incapaz de mantener su status actual.

Se ha evidenciado que la IP por enfermedad común está relacionada con las características sociodemográficas ${ }^{4}$ y las condiciones de trabajo en un sentido pronóstico ya que, aunque el problema de salud por el que se concede la IP no sea de origen laboral, la población afectada podrá o no seguir trabajando dependiendo de las condiciones de su puesto de trabajo ${ }^{5}$. Conocer estas variables es de gran utilidad para adecuar programas preventivos sobre la población susceptible, lo que requiere una actuación a varios niveles, con la implicación de las administraciones autonómicas, locales y las empresas.

Al objeto de adecuar las medidas preventivas desde un Servicio de Prevención y Salud Laboral en una empresa de la Administración Local se ha procedido a llevar a cabo el presente estudio cuyo objetivo es describir las características de la población trabajadora de la Diputación Provincial de Cádiz a los que se les ha concedido la IP en el periodo 1995-2009.

\section{MÉTODOS}

Ámbito

El estudio se ha realizado en una empresa pública de competencias y ámbito de actuación provincial con una población media de 1600 trabajadores/as, que tiene constituido un Servicio de Prevención y Salud Laboral propio, al amparo de lo recogido en la Ley de Prevención de Riesgos Laborales ${ }^{6}$.

Como empresa pública de la Administración Local sus competencias son muy diversas y se distribuyen en más de un centenar de centros de trabajo. 


\section{Diseño}

Para conseguir los objetivos descritos se ha llevado a cabo un estudio descriptivo retrospectivo.

\section{Población y muestra}

La población a estudio está compuesta por toda la población trabajadora de la empresa anteriormente descrita a la que se le haya concedido una IP por el Equipo de Valoración de Incapacidades de la Dirección Provincial del Instituto Nacional de la Seguridad Social de Cádiz en el periodo del 1 de enero de 1995 a 31 de diciembre de 2009. Se excluyeron aquellos casos correspondientes a revisión de grado, es decir, los que teniendo ya reconocida la incapacidad se reevaluaron para determinar la posible variación en el grado de ésta.

Por el número de casos y la accesibilidad a la información se decidió llevar a cabo un estudio poblacional exhaustivo de todos los individuos que cumplieron los criterios de inclusión anteriormente descritos.

\section{Operacionalización de variables}

Para el presente estudio se han considerado las siguientes variables:

- Variables sociodemográficas:

- Sexo.

- Edad.

- Antigüedad en la empresa.

- Tipo de trabajo desempeñado en el momento de la resolución de la IP. Debido a la gran diversidad de ocupaciones no pudo encontrarse en la literatura una clasificación que se adecuara a ellas. Por tanto, se diseñó una categorización ad hoc que contenía cinco grupos en función de las características del trabajo realizado: manual, administrativo y afines, gestión, socio-sanitario y/o asistencial, y educativo. Los puestos de trabajo que contienen cada grupo pueden consultarse en la tabla 1.

- Vinculación laboral: Personal funcionario o laboral.

- Variables del proceso de incapacidad:

- Grado de Incapacidad: Parcial, Absoluta, Total o Gran Invalidez.

- Tipo de patología principal causante de la situación incapacitante. Se utilizó para su codificación la Clasificación Internacional de Enfermedades-9 $\mathrm{MC}^{7}$.

- Número de Incapacidades Laborales Transitorias (IT) en los cinco años previos a la IP.

- Número de días de IT en los cinco años previos a la IP.

Tabla 1. Clasificación según el tipo de trabajo realizado

\begin{tabular}{|c|c|c|c|c|}
\hline \multicolumn{5}{|c|}{ Tipo de trabajo } \\
\hline $\begin{array}{c}\text { Principalmente } \\
\text { manual }\end{array}$ & $\begin{array}{l}\text { Principalmente } \\
\text { administrativo } \\
\text { y afines }\end{array}$ & $\begin{array}{c}\text { Principalmente } \\
\text { de gestión }\end{array}$ & $\begin{array}{l}\text { Principalmente } \\
\text { socio-sanitario } \\
\text { y/o asistencial }\end{array}$ & $\begin{array}{c}\text { Principalmente } \\
\text { educativo }\end{array}$ \\
\hline - Albañil & - Administrativo & - Encargado & - Auxiliar clínica & - Auxiliar de Aula \\
\hline - Ayudante & - Ayudante de & - Jefe de & - Auxiliar geriátrico & - Educador \\
\hline de Cocina & Recaudación & Negociado & - Auxiliar & - Instructora \\
\hline - Ayudante & - Delineante & - Perito Agrícola & psiquiátrico & - Maestro \\
\hline de Servicios & - Oficial calculista & - Perito Industrial & - Cuidador & \\
\hline Especiales & & & & \\
\hline - Carpintero & & & & \\
\hline
\end{tabular}




\begin{tabular}{|c|c|c|c|c|}
\hline \multicolumn{5}{|c|}{ Tipo de trabajo } \\
\hline $\begin{array}{c}\text { Principalmente } \\
\text { manual }\end{array}$ & $\begin{array}{c}\text { Principalmente } \\
\text { administrativo } \\
\mathrm{y} \text { afines }\end{array}$ & $\begin{array}{l}\text { Principalmente } \\
\text { de gestión }\end{array}$ & $\begin{array}{l}\text { Principalmente } \\
\text { socio-sanitario } \\
\text { y/o asistencial }\end{array}$ & $\begin{array}{l}\text { Principalmente } \\
\text { educativo }\end{array}$ \\
\hline $\begin{array}{l}\text { - Cocinero } \\
\text { - Conductor } \\
\text { - Costurera } \\
\text { - Electricista } \\
\text { - Fontanero } \\
\text { - Herrero } \\
\text { - Jardinero } \\
\text { - Lavandera } \\
\text { - Limpiadora } \\
\text { - Maquinista } \\
\text { - Mecánico } \\
\text { - Oficial } \\
\text { de Cocina } \\
\text { - Oficial } \\
\text { polivalente } \\
\text { - Peón } \\
\text { - Peón agrícola } \\
\text { - Peón Caminero } \\
\text { - Personal } \\
\text { trabajador de } \\
\text { mantenimiento } \\
\text { - Pintor } \\
\text { - Vigilante }\end{array}$ & $\begin{array}{l}\text { - Oficial de la } \\
\text { Administración } \\
\text { Especial } \\
\text { - Oficial de } \\
\text { Mayordomía } \\
\text { - Oficial de } \\
\text { Recaudación } \\
\text { - Ordenanza } \\
\text { - Técnico de } \\
\text { video } \\
\text { - Telefonista }\end{array}$ & $\begin{array}{l}\text { - Recaudador } \\
\text { - Técnico } \\
\text { de Cultura } \\
\text { - Técnico } \\
\text { de Gestión } \\
\text { - Técnico de la } \\
\text { administración } \\
\text { General } \\
\text { - Técnico de } \\
\text { Grado Medio } \\
\text { - Viceinterventor }\end{array}$ & $\begin{array}{l}\text { - Enfermero/a } \\
\text { - Médico } \\
\text { - Psicólogo/a } \\
\text { - Trabajadora } \\
\quad \text { Social }\end{array}$ & $\begin{array}{l}\text { - Profesor de } \\
\text { Formación } \\
\text { Profesional } \\
\text { - Profesor de } \\
\text { Educación } \\
\text { Física }\end{array}$ \\
\hline
\end{tabular}

\section{Fuente de datos}

Los datos se recogieron de los archivos del Departamento de Personal y Recursos Humanos, así como de las historias clínicas y expedientes existentes en el Servicio de Prevención y Salud Laboral.

\section{Análisis de datos}

Se realizó una base de datos en el paquete informático SPSS v.17.0 con el que además se realizó el análisis estadístico. Se llevó a cabo primeramente un análisis descriptivo de los datos en el que las variables cualitativas se expresaron en forma de frecuencias y porcentajes y las cuantitativas como media y desviación estándar.

Para analizar la asociación entre las variables categóricas se utilizó la prueba de la chi cuadrado o el test exacto de Fisher. Para establecer la relación entre las variables cuantitativas con variables cualitativas se utilizaron la prueba t de Student o el test no paramétrico de Wilcoxon en el caso de que la variable cualitativa fuera dicotómica, y el análisis de la varianza (ANOVA) o el test no paramétrico de Kruskal-Wallis en el caso de que la variable cualitativa fuera politómica. Previo a la aplicación de unos u otros de los estadísticos anteriores se exploró la normalidad de las variables con la prueba de Kolmogorov-Smirnov. Todos los resultados se consideraron significativos con una $\mathrm{p}<0,05$.

\section{Consideraciones éticas}

El presente estudio cumple todas las premisas éticas consensuadas en la Declaración de Helsinki y las legales establecidas por la legislación vigente. Se consideró especialmente la protección de confidencialidad de los datos de los trabajadores/as y se obtuvieron los permisos necesarios de estos y de la institución para acceder a los mismos. 


\section{RESULTADOS}

El número total de IP concedidas en el periodo estudiado fue de 192, con la distribución por años que se presenta en el gráfico 1 . De ellas el 50,5\% se concedieron en grado de Total, el 49,7 \% en Absolutas y un 1,6 \% en Gran Invalidez. En la tabla 2 se exponen los resultados de las variables que recogen características sociodemográficas de los trabajadores y trabajadoras a los que les fue concedida la IP y en la tabla 3 las relativas al propio proceso.

Tabla 2. Resultados relativos a las características Sociodemográficas

\begin{tabular}{|c|c|c|c|c|c|}
\hline & & \multicolumn{2}{|c|}{$\mathbf{F}$} & \multicolumn{2}{|c|}{$\%$} \\
\hline \multirow{3}{*}{ Sexo } & Hombre & \multicolumn{2}{|c|}{90} & \multicolumn{2}{|c|}{46,9} \\
\hline & Mujer & \multicolumn{2}{|c|}{102} & \multicolumn{2}{|c|}{53,1} \\
\hline & Total & \multicolumn{2}{|c|}{192} & \multicolumn{2}{|c|}{100,0} \\
\hline \multirow{3}{*}{ Vinculación laboral } & Funcionario & \multicolumn{2}{|c|}{115} & \multicolumn{2}{|c|}{60,8} \\
\hline & Laboral & \multicolumn{2}{|c|}{74} & \multicolumn{2}{|c|}{39,2} \\
\hline & Total & \multicolumn{2}{|c|}{192} & \multicolumn{2}{|c|}{100,0} \\
\hline \multirow{6}{*}{ Tipo de Trabajo } & Manual & \multicolumn{2}{|c|}{86} & \multicolumn{2}{|c|}{44,8} \\
\hline & Administrativo y afines & \multicolumn{2}{|c|}{43} & \multicolumn{2}{|c|}{22,4} \\
\hline & Gestión & \multicolumn{2}{|c|}{10} & \multicolumn{2}{|c|}{5,2} \\
\hline & Socio-sanitario y/o asistencial & \multicolumn{2}{|c|}{32} & \multicolumn{2}{|c|}{16,7} \\
\hline & Educativo & \multicolumn{2}{|c|}{21} & \multicolumn{2}{|c|}{10,9} \\
\hline & \multirow[t]{2}{*}{ Total } & \multicolumn{2}{|c|}{192} & \multicolumn{2}{|c|}{100,0} \\
\hline & & Mínimo & Máximo & Media & $\mathrm{DE}$ \\
\hline \multicolumn{2}{|c|}{ Edad de concesión de la IP } & 30 & 70 & 54,06 & 7,014 \\
\hline \multicolumn{2}{|c|}{ Años de antigüedad en la empresa } & 3 & 46 & 24,98 & 7,813 \\
\hline
\end{tabular}

Tabla 3. Resultados relativos al proceso de IP

\begin{tabular}{|c|c|c|c|c|c|c|c|c|c|}
\hline & & \multicolumn{2}{|c|}{$\begin{array}{l}\text { Incapacidad } \\
\text { Total }\end{array}$} & \multicolumn{2}{|c|}{$\begin{array}{c}\text { Incapacidad } \\
\text { Absoluta }\end{array}$} & \multicolumn{2}{|c|}{$\begin{array}{c}\text { Gran } \\
\text { Invalidez }\end{array}$} & \multicolumn{2}{|c|}{ Total } \\
\hline & & $\mathbf{F}$ & $\%$ & $\mathbf{F}$ & $\%$ & $\mathbf{F}$ & $\%$ & $\mathbf{F}$ & $\%$ \\
\hline \multirow{12}{*}{$\begin{array}{l}\text { Enfermedades/ } \\
\text { Lesiones }\end{array}$} & Infecciosas y Parasitarias & 1 & 1,1 & 0 & 0 & 0 & 0 & 1 & 0,5 \\
\hline & Neoplasias & 35 & 38 & 10 & 10,3 & 0 & 0 & 45 & 23,4 \\
\hline & $\begin{array}{l}\text { Sangre y Órganos } \\
\text { Hematopoyéticos }\end{array}$ & 2 & 2,2 & 0 & 0 & 0 & 0 & 2 & 1,0 \\
\hline & Trastornos Mentales & 20 & 21,7 & 14 & 14,4 & 2 & 66,7 & 36 & 18,8 \\
\hline & Oftalmológicas & 3 & 3,3 & 1 & 1 & 0 & 0 & 4 & 2,1 \\
\hline & Sistema Circulatorio & 13 & 14,1 & 15 & 15,5 & 1 & 33,3 & 29 & 15,1 \\
\hline & Aparato Respiratorio & 4 & 4,3 & 4 & 4,1 & 0 & 0 & 8 & 4,2 \\
\hline & Aparato. Digestivo & 4 & 4,3 & 2 & 2,1 & 0 & 0 & 6 & 3,1 \\
\hline & Piel y Tejido. Subcutáneo & 2 & 2,2 & 1 & 1 & 0 & 0 & 3 & 1,6 \\
\hline & $\begin{array}{l}\text { Sistema Osteomioarticular } \\
\text { y Tejido Conectivo }\end{array}$ & 8 & 8,7 & 49 & 50,5 & 0 & 0 & 57 & 29,7 \\
\hline & Aparato. Genitourinario & 0 & 0 & 1 & 1 & 0 & 0 & 1 & 0,5 \\
\hline & Total & 92 & 100 & 97 & 100 & 3 & 100 & 192 & 100 \\
\hline & & \multicolumn{2}{|c|}{ Mínimo } & \multicolumn{2}{|c|}{ Máximo } & \multicolumn{2}{|c|}{ Media } & \multicolumn{2}{|c|}{$\mathrm{DE}$} \\
\hline \multicolumn{2}{|c|}{ Número de procesos de ILT en los 5 años previos a la IP } & \multicolumn{2}{|c|}{1} & \multicolumn{2}{|c|}{16} & \multicolumn{2}{|c|}{5,09} & \multicolumn{2}{|c|}{2,98} \\
\hline \multicolumn{2}{|c|}{ Días de ILT en los 5 años previos a la IP } & \multicolumn{2}{|c|}{12} & \multicolumn{2}{|c|}{1341} & \multicolumn{2}{|c|}{541,41} & \multicolumn{2}{|c|}{256,80} \\
\hline
\end{tabular}


Gráfico 1. N. ${ }^{\circ}$ de resoluciones de IP por años

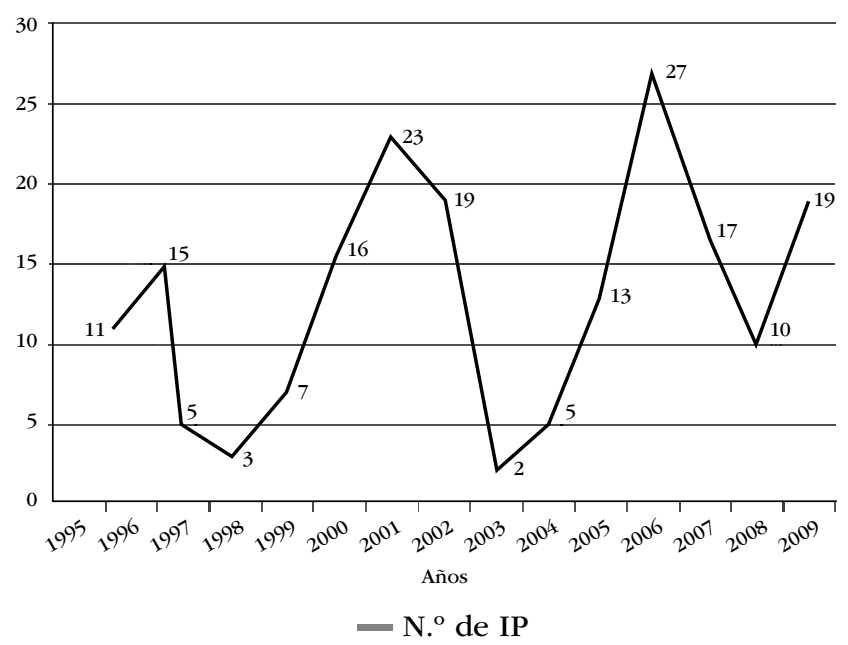

El tipo de patología causante de la IP se asocia significativamente con el sexo $(\mathrm{p}=0,006)$, el tipo de trabajo desempeñado en el momento de la resolución de la IP $(\mathrm{p}=0,007)$, la edad en la que se concede $(\mathrm{p}<0,001)$ y con el grado de IP asignada $(\mathrm{p}<0,000)$.

El número de IT en los cinco años previos a la IP no se asocia de manera significativa a ninguna de las variables estudiadas y los días de baja por IT en dicho periodo únicamente al tipo de trabajo desempeñado en el momento de la resolución de la IP $(\mathrm{p}=0,002)$.

\section{DISCUSIÓN}

A la cohorte estudiada se le concedió la IP con una media de 54,06 años de edad y acumulaba un total de 24,98 años trabajados, predominando en ésta el sexo femenino, la vinculación laboral como funcionario y los trabajos de tipo manual.

El número de IP concedidas por año en el periodo estudiado sigue un patrón oscilante sin una tendencia lógica aparente, a diferencia de los datos obtenidos de estadísticas nacionales en las que se evidencia una tónica ascendente continua en la última década ${ }^{8}$. La mayor parte de las IP fueron concedidas con grado de total, al igual que a nivel de todo el país en el periodo que contempla el estudio, donde dicho grado sigue una progresión anual ascendente desde el año $1998^{9}$. No obstante, estas diferencias y similitudes pueden considerarse anecdóticas ya que derivan de la comparación de una población numerosa y heterogénea con otra reducida y homogénea, sin embargo ha sido imposible encontrar en la literatura trabajos que estudien poblaciones laborales de características similares.

Las enfermedades músculo-esqueléticas son las que más frecuentemente derivan en IP en la población estudiada. La mayor parte de los trabajadores/as con esta causa de IP desarrollaba actividades de tipo manual, socio-sanitario y/o asistencial, predominando dentro de este último grupo el puesto de auxiliar geriátrico, que tiene como cometido destacado la movilización de pacientes. Esto puede poner en evidencia que, aunque las condiciones de trabajo no tengan relación en un sentido etiológico con la enfermedad, sí puede tenerla con el pronóstico ${ }^{10}$ lo que determina la IP. Es de destacar que el segundo lugar en cuanto a producción de IP es ocupado por las enfermedades neoplásicas, que rondan la cifra del $20 \%$ del total. Este dato es similar al obtenido en un estudio anterior ${ }^{11}$ que evaluaba la misma población en otro periodo de tiempo, lo que apoya una estabilidad en la prevalencia de IP de origen neoplásico. El tercer lugar es ocupado por los trastornos mentales. Esta distribución etiológica de IP es compartida por otros trabajos similares en poblaciones más numerosas ${ }^{12}$. 
Tanto las neoplasias como las enfermedades músculo-esqueléticas son causa de IP en el grupo de edad de entre los 50 y los 59 años mientras que en los trastornos mentales el intervalo se adelanta a la década de los 40, algo razonable si consideramos que más del $50 \%$ de los casos de trastornos del ánimo y ansiosos, patologías incapacitantes en sus casos más graves y de elevada incidencia ${ }^{13}$, suelen iniciarse en el rango de edad de los $25-45^{14}$. Respecto al grado de incapacidad señalar que las enfermedades neoplásicas son las que más se califican como absolutas, mientras que las del sistema músculo-esquelético son mayoritariamente causa de IP total. Esto puede deberse a la mayor repercusión sistémica de las primeras respecto a las segundas, en las que un alto porcentaje afectan a áreas concretas del organismo lo que incapacitaría al individuo para su trabajo habitual pero no para otros trabajos, o bien al hecho de que el diagnóstico de neoplasia sea un condicionante que determine a priori el otorgar ese grado de incapacidad. Las enfermedades mentales se reparten entre uno y otro tipo debido a la gran variabilidad de repercusión en el desempeño laboral de las distintas entidades que conforman dicho grupo.

Lo anteriormente expuesto justifica la asociación hallada entre la patología causante de la IP con el tipo de trabajo desempeñado en el momento de la resolución, la edad a la que se concede y el grado de IP asignada. La relación además con el sexo es razonable si se considera las diferencias existentes en la distribución de las patologías por género ${ }^{12}$.

Es necesario subrayar el número elevado de bajas y de jornadas perdidas en los cinco años precedentes a la IP. Estos ascienden a una media de 5,09 bajas por IT y 108 días por trabajador/a. Sin embargo, el número de bajas no se asocia con ninguna de las variables estudiadas y los días de IT únicamente con el tipo de trabajo desarrollado, y no con el tipo de patología causante de la IP como cabría esperar. Esto sugiere que la concesión de IT es un proceso complejo en el que intervienen otros factores, algunos externos y otros internos al individuo, no considerados en este estudio. Así mismo, no es posible descartar la influencia en la asociación hallada de la agrupación de puestos de trabajo realizada ad boc por los autores y el haber considerado exclusivamente los 5 años previos a la IP por la imposibilidad de acceso a los datos necesarios para ampliar el periodo de seguimiento. Otra de las limitaciones la constituye la dificultad de acceso a los diagnósticos de los procesos de IT previos a la IP del personal acogido al régimen general de la Seguridad Social y con Asistencia Sanitaria con cargo al Sistema Sanitario Público Andaluz, ya que estos no están recogidos en los archivos clínicos, al ser el responsable del proceso asistencial el médico de Atención Primaria y no el médico del Servicio de Salud Laboral.

Este estudio aporta indicios sobre la frecuencia y distribución de las variables sociodemográficas y propias de la IP en una empresa pública que alcanza un considerable número de expedientes resueltos en un periodo de 15 años. Esto permite ir descifrando algunos de los aspectos susceptibles de ser abordados desde el punto de vista de la prevención que redunde, no sólo en una mejora de la salud de los trabajadores, sino también en la sostenibilidad de una sociedad en progresivo envejecimiento e incremento de los problemas de salud crónicos.

\section{AGRADECIMIENTOS}

A la Excelentísima Diputación Provincial de Cádiz por las facilidades proporcionadas para la realización del estudio.

\section{REFERENCIAS BIBLIOGRÁFICAS}

1. Ley General de la Seguridad Social. Real Decreto legislativo 1/1994 (20 junio 1994).

2. Álvarez de la Rosa M. Invalidez permanente y Seguridad Social. Madrid: Civitas; 1982. 305 p. 
MEDICINA

3. Instituto Nacional de Estadística. Pensiones contributivas del sistema de la Seguridad Social. Serie 19942009. Madrid: Instituto Nacional de Estadística, 2009 [citado 1 de julio de 2011]. Disponible en: http:// www.ine.es/jaxi/tabla.do?type=pcaxis \&path=/t25/a072/a01/10/\&file=c50002.px

4. Minkler M, Fuller-Thomson E, Guralnik JM. Gradient of disability across the socioeconomic spectrum in the United States. N Engl J Med 2006; 355(7):695-703.

5. Laine S, Gimeno D, Virtanen M, Oksanen T, Vahtera J, Elovainio M, et al. Job strain as a predictor of disability pension: the Finnish Public Sector Study. J Epidemiol Commun Health 2009 January 1, 2009; 63(1):24-30.

6. Ley de Prevención de Riesgos Laborales. Ley 31/1995 (8 noviembre 1995).

7. Ministerio de Sanidad y Consumo. Clasificación internacional de enfermedades 9. ${ }^{a}$ revisión, modificación clínica (CIE 9-MC) [edición electrónica]. 5. . ed. Madrid: Ministerio de Sanidad y Consumo, 2006 [citado 15 de junio de 2010]. Disponible en la World Wide Web: http://www.msc.es/estadEstudios/ecie9mc/ webcie9mc/webcie9mc.htm.

8. Ministerio de Trabajo e Inmigración. Evolución mensual de las pensiones del sistema de la Seguridad Social. Avance Agosto de 2010 [online]. Madrid: Ministerio de Trabajo e Inmigración; 2010 [citado 15 de junio de 2010]. Disponible en la World Wide Web: http://www.la-moncloa.es/NR/rdonlyres/DB1DC0B8B5AF-4DC1-98FB-C9B951176319/114688/AVANCEPENSIONESAGOSTO20103.pdf.

9. Instituto Nacional de Estadística. Pensiones del sistema de la Seguridad Social. [online]. Madrid: Instituto Nacional de Estadística, 2009 [citado 1 de abril de 2011]. Disponible en la World Wide Web: http://www.ine.es/jaxi/tabla.do?type=pcaxis\&path=/t25/a072/a01/10/\&file=c50002.px

10. Benavides FG, Durán X, Martínez JM, Jódar P, Boix P, Amable M. Incidencia de incapacidad permanente en una cohorte de trabajadores afiliados a la Seguridad Social, 2004-2007. Gac Sanit 2010; 24(5):385-90.

11. Ares Camerino A, Sainz Vera B, Marchena Aparicio JC, Soto Pino ML. Las enfermedades neoplásicas como causa de la incapacidad permanente. Med Segur Trab 2008; 54(210):57-62.

12. Arancón Viguera A. Estudio epidemiológico sobre la incapacidad permanente para el trabajo. Med Gen (Madr). 2002; 45:462-70.

13. Martínez M, Dolz M, Alonso J, Luque I, Palacín C, Bernal M, et al. Prevalencia de los trastornos mentales y factores asociados: resultados del estudio ESEMeD-España. Med Clin (Barc). 2006; 126 (12):445-51.

14. Kessler RC, Amminger GP, Aguilar-Gaxiola S, Alonso J, Lee S, Ustün TB. Age of onset of mental disorders: a review of recent literature. Curr Opin Psychiatry. 2007; 20(4):359-64. 